\title{
Anticipated synchronization in neuronal systems subject to noise
}

\author{
Raúl Toral ${ }^{a, b}$, Claudio R. Mirasso ${ }^{b}$, C. Masoller ${ }^{c}$, M. Ciszak ${ }^{a}$ and O. Calvo ${ }^{b}$ \\ ${ }^{a}$ Instituto Mediterráneo de Estudios Avanzados (IMEDEA) CSIC-UIB, \\ Ed. Mateu Orfila, Campus UIB, 07122-Palma de Mallorca, Spain \\ ${ }^{b}$ Departament de Física, Universitat de les Illes Balears, \\ Campus UIB, 07122-Palma de Mallorca, Spain \\ ${ }^{c}$ Facultad de Ciencias, Universidad de la República, Igua 4225, Montevideo 11400, Uruguay
}

\begin{abstract}
We report the observation of synchrony in two unidirectionally coupled (master-slave) model neurons (implemented by electronic circuits) in a noisy environment. Both neurons are subjected to the same random stimulus, and there is a recurrent inhibitory delayed connection in the slave neuron. We observe that synchrony occurs shifted in time, such that the slave neuron anticipates, i.e., forecasts, the response of the master neuron. By incorporating the effects of unidirectional coupling, delayed feedback and common noise into models of two spiking neurons, we are able to simulate successfully the experimental observations.
\end{abstract}

Keywords: Synchronization, time delay dynamics, excitability, noise.

\section{INTRODUCTION}

Neurons, as well as cardiac tissue, are classical prototypes of excitable systems: their response to an external perturbation is highly non-linear and depends on its magnitude and timing: if the perturbation is small, the system evolves back to the steady state; but if the perturbation exceeds a certain threshold, the system fires a pulse-like spike (action potential). Following the onset of the excitation, there is an interval (refractory period) during which another perturbation does not induce a new pulse. Sensory neurons transform environmental stimuli into trains of action potentials, usually referred to as 'spikes'. The response of a sensory neuron to different stimuli can cause the firing of such a spike. The information about the external stimuli is transmitted into the brain using a code based on the time intervals between spikes. Different regions of the brain must communicate with each other in order to integrate the information into a global picture. This global process may lead to a production of simultaneous spikes yielding a synchronized state between neurons. In the last years, it has been proposed that synchronous neuronal oscillations underlie many cortical processes, ${ }^{1-4}$ and it has been postulated that some cortical structures are able to predict the most likely input several milliseconds ahead. ${ }^{5-7}$

Real neurons, as well as the heart, are complicated non-linear systems involving a large number of variables. Nevertheless, the essential features of their excitable behavior can be captured with a much-reduced description. The FitzHugh-Nagumo (FHN) and the Hodgkin-Huxley (HH) models provide two of the simplest representation of firing dynamics and they have been widely used as a prototypic models for both, spiking neurons and cardiac cells. $^{8,9}$ Here we study the synchronization of two identical FitzHugh-Nagumo and Hudgkin-Huxley neurons, unidirectionally coupled, in the presence of a common noisy environment, and when the slave neuron has its own delayed feedback mechanism (see the schematic diagram shown in Fig. 1).

Further author information: Send correspondence to R. Toral, e-mail: raul@imedea.uib.es, http://www.imedea.uib.es 


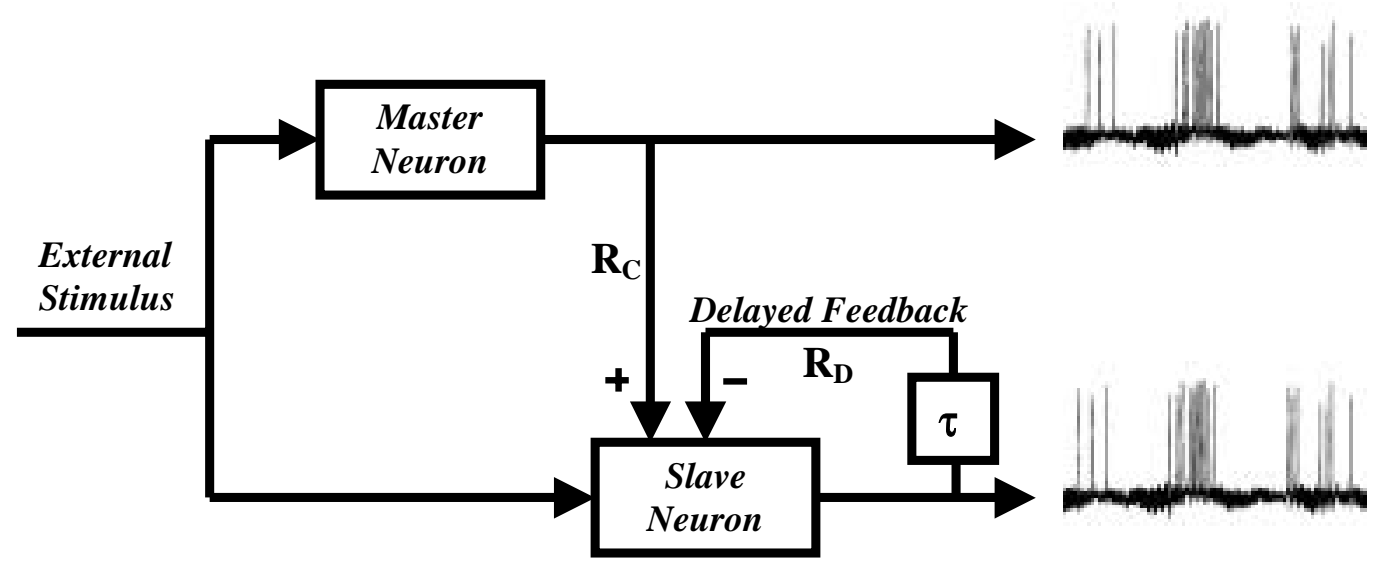

Figure 1. Schematic diagram of two neurons coupled in a unidirectional configuration, subjected to the same external stimulus and with a time delayed feedback in the slave neuron.

\section{EXPERIMENTAL RESULTS}

We have built an electronic circuit that emulates two FN neurons driven with Gaussian white noise and coupled as shown in Fig. 1. The circuit was constructed from standard electronic components (operational amplifiers and analog multipliers). Two identical (within components tolerance) neurons were implemented (see Fig. 2). The neurons were built using operational amplifiers and the cubic non-linearity described by $x(x-a)(x-b)($ see eq. (1) below) was implemented using analog multipliers (AD633) in a circuit not shown for simplicity. ${ }^{10}$ The resistance $R_{C}$ controls the strength of the unidirectional coupling between the master and the slave neurons. The resistance $R_{D}\left(R_{D}=R_{C}\right.$ in our case) controls the strength of the delayed feedback into the slave neuron. The coupling and the delayed feedback have opposite signs: while the master signal was obtained at point $B$ of Fig. 2, where the voltage is $-V_{m}$, the slave signal that goes into the delay line was obtained at point $C$ of Fig. 2, where the voltage is $+V_{s}$. The different signs are due to the inverters that are located in between points $A$ and $B$ and $C$ and $D$. The threshold on both neurons was controlled by a potentiometer represented by its equivalent circuit: offset and $R_{0}$. The analog delay line for the delayed feedback in the slave neuron was built using bucket brigade circuits (MN3004). A function generator with white noise output capabilities (HP33120A) was used to excite both neurons. The signals were acquired using LabView and National Instruments DAQ 6025E data acquisition board. Similar variants of this circuit have been previously used to model the response of various types of neurons. ${ }^{11}$

We show the existence of an anticipated synchronization mechanism ${ }^{12}$ by which a neuron might be able to predict the future response of another neuron. The mechanism responsible for the anticipation is interesting for two main reasons: (i) the master neuron is unaware of the presence of the slave neuron and its own dynamics is not altered; (ii) due to the random stimulus the master neuron produces a train of spikes that is unpredictable.

For an appropriate value of the coupling, represented in the circuit by a resistance $R_{C}$ (see also Fig. 1), we observe that, after a transient, the master and slave neurons synchronize in such a way that the slave neuron anticipates the fires of the master neuron by a time interval approximately equal to the delay time $\tau$ of the synaptic feedback mechanism. Fig. 3 (a) shows a typical spike train while Fig. 3 (b) displays in detail a single spike. ${ }^{13}$ We observe that the firings of the master and the slave neurons start at about the same time: anticipation begins during the rising of the peak and it vanishes when the neurons are in the unexcited state. In other words, anticipation is a local process triggered during the firing of the spikes. The anticipation is due to the combined effect of unidirectional coupling (the synaptic connection controlled by $R_{C}$ in Fig. 1 ) and negative delayed feedback (the synaptic delayed connection controlled by a resistance $R_{D}$ in Fig. 1). Without coupling and feedback $\left(R_{C}=R_{D}=0\right)$ the neurons fire pulses which are, in general, desynchronized (due to the small mismatch between the circuits). 


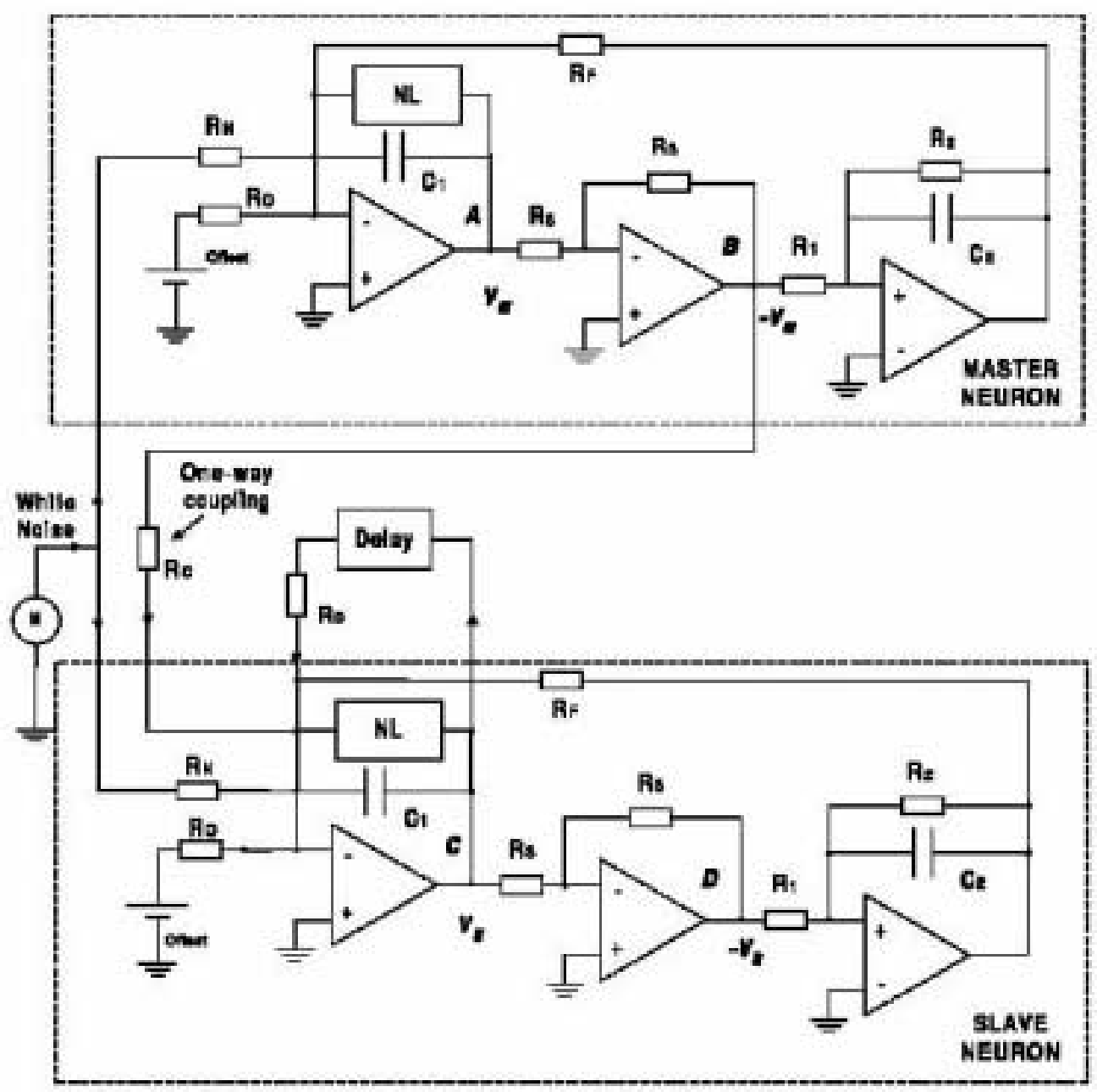

Figure 2. Figure 2. Circuit implementation of two coupled neurons. $\mathrm{R} 1=125 \mathrm{k} \Omega, \mathrm{R} 2=50 \mathrm{k} \Omega, \mathrm{R} 3=10 \mathrm{k} \Omega, \mathrm{RC}=\mathrm{RD}=100$ $\mathrm{k} \Omega, \mathrm{RF}=10 \mathrm{k} \Omega, \mathrm{RN}=10 \mathrm{k} \Omega, \mathrm{RO}=10 \mathrm{k} \Omega, \mathrm{C} 1=100 \mathrm{nF}, \mathrm{C} 2=1 \mathrm{mF}$.

We observe that the slave neuron occasionally makes an error in anticipating the master firings (notice that in Fig. 3 there is such an error at the end of the spike train of the slave neuron). The longer the anticipation time $\tau$, the larger the number of errors, and for $\tau$ long enough synchrony is lost. Even though occasionally the slave neuron fires a pulse without a firing of the master, the opposite behavior never occurs: for each pulse fired by the master neuron there is a corresponding anticipated pulse fired by the slave neuron.

Anticipation in the synchronization of chaotic systems is a subject that has received a lot of attention recently. ${ }^{11,14-16}$ While the anticipated behavior in the case of a master system with its own delayed feedback is well understood, the mechanism underlying the anticipation remains elusive when the master system is memoryless (i.e., without a feedback loop). The present paper reports the first experimental observation of anticipation in one-way coupled systems in which the master system does not have a delayed feedback mechanism, and it is unaware of the presence of the slave system. 


\section{NUMERICAL RESULTS}

To further investigate the synchronization behavior observed in our experiments, we numerically simulate an extension of the FitzHugh-Nagumo model ${ }^{8,9}$ that takes into account unidirectional coupling, common external stimulus, and delayed negative feedback in the slave neuron. The model equations are

$$
\begin{aligned}
\frac{d x_{m}}{d t} & =-x_{m}\left(x_{m}-a\right)\left(x_{m}-1\right)-y_{m}+f_{o}+\xi(t) \\
\frac{d y_{m}}{d t} & =\epsilon\left(x_{m}-b y_{m}\right) \\
\frac{d x_{s}}{d t} & =-x_{s}\left(x_{s}-a\right)\left(x_{s}-1\right)-y_{s}+K\left[x_{m}(t)-x_{s}(t-\tau)\right] \\
& +f_{o}+\xi(t) \\
\frac{d y_{s}}{d t} & =\epsilon\left(x_{s}-b y_{s}\right),
\end{aligned}
$$

where $x_{m}, y_{m}\left(x_{s}, y_{s}\right)$ are the fast and slow variables of the master (slave) neuron, $\xi(t)$ is the common external stimulus, $a, b, \epsilon$ and $f_{o}$ are constants. $K$ is the strength of the synaptic connection and $\tau$ is the delay of the synaptic feedback mechanism of the slave neuron. Notice that the dynamics of the master neuron is independent of that of the slave neuron, i.e., it is a one-way coupling. Notice also that the synaptic feedback term, $x_{s}(t-\tau)$, has the opposite sign to the synaptic coupling term, $x_{m}(t)$.

The results of our simulations, when using the Fitzhugh-Nagumo model are shown in Figs. 4a, 4b. The parameters we use are $a=0.139, b=2.54, \epsilon=0.008, f_{o}=0.03, K=0.03, \tau=10$. The external stimulus $\xi$ is a Gaussian white noise with zero mean and correlation $\left\langle\xi(t) \xi\left(t^{\prime}\right)\right\rangle=2 D \delta\left(t-t^{\prime}\right)$ with $D=2.45 \times 10^{-5}$. The numerical results mimic those of the experiments.

We have also performed simulations with a more realistic model, namely the model of electro-receptors proposed by Braun et. al. ${ }^{17-19}$ This model is a modification of the Hodgkin-Huxley (HH) neuron model:

$$
C_{M} \frac{d V}{d t}=-I_{N a}-I_{K}-I_{s d}-I_{s r}-I_{l}+\xi(t)
$$

where $V$ is the potential voltage across the membrane and $C_{M}$ is the capacitance; $I_{N a}$ and $I_{K}$ are the fast sodium and potassium currents that generate the action potential (de- and repolarizing currents), $I_{s d}$ and $I_{s r}$ are additional slow currents, $I_{l}$ is a passive leak current and $\xi(t)$ is the external stimulus. The voltage dependent currents are modelled as ${ }^{17-19}$ :

$$
\begin{aligned}
I_{x} & =\rho g_{x} a_{x}\left(V-V_{x}\right) \\
I_{l} & =g_{l}\left(V-V_{l}\right),
\end{aligned}
$$

where subindex $x$ refers to $N a, K, s r$ and $s d ; \rho$ is a temperature dependent factor; $g_{x}, g_{l}$ the maximum conductance and $V_{x}, V_{l}$ the reverse potentials. The activation variable $a_{x}$ are described by the dynamical equations

$$
\frac{d a_{x}}{d t}=\frac{\phi\left(a_{x, \infty}-a_{x}\right)}{\tau_{x}}
$$

where $x$ refers to $K$ and $s d ; \phi$ is a temperature like factor, $\tau_{x}$ are the time constants, $a_{x, \infty}$ are steady state activations, while

$$
\frac{d a_{s r}}{d t}=\frac{-\phi\left(\eta I_{s d}+\kappa a_{s r}\right)}{\tau_{s r}}
$$

with $\eta$ and $\kappa$ constant. For more details and parameter values see Refs.. ${ }^{17-19}$ 
We extend the model to account for unidirectional coupling, delayed feedback and common noise, in the same way as in the FHN model, in eq. 5. The new equations are:

$$
\begin{aligned}
C_{M} \frac{d V_{m}}{d t} & =-I_{N a}^{m}-I_{K}^{m}-I_{s d}^{m}-I_{s r}^{m}-I_{l}^{m}+\xi(t) \\
C_{M} \frac{d V_{s}}{d t} & =-I_{N a}^{s}-I_{K}^{s}-I_{s d}^{s}-I_{s r}^{s}-I_{l}^{s}+\xi(t) \\
& +K\left[V_{m}(t)-V_{s}(t-\tau)\right] .
\end{aligned}
$$

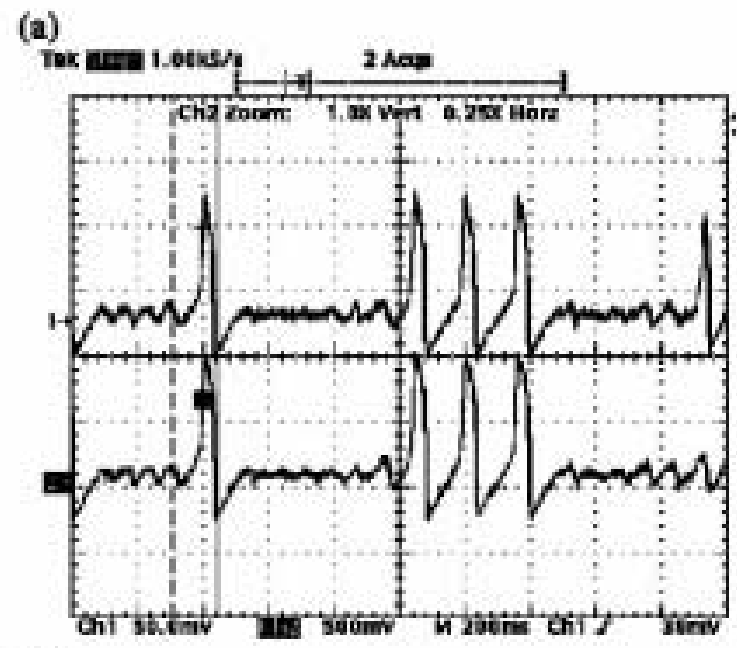

(b)

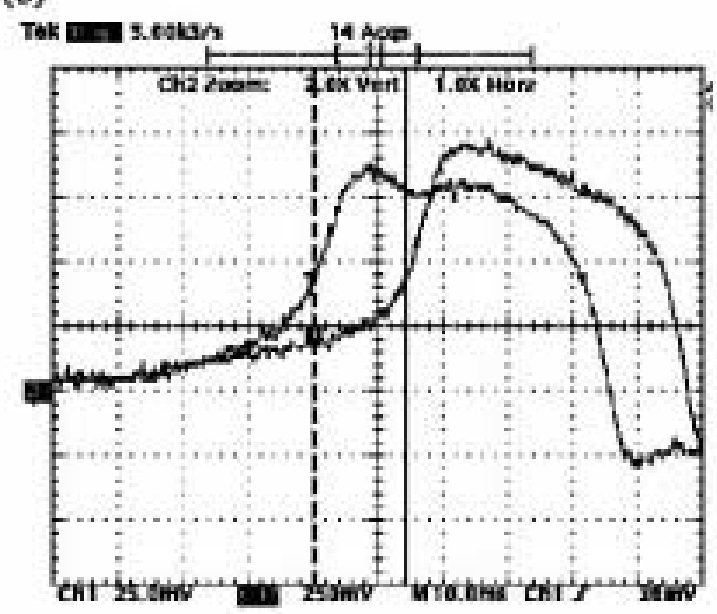

Figure 3. (a) Experimental train of spikes that shows anticipation in the spikes fired by the slave neuron (upper trace) with respect to the spikes fired by the master neuron (lower trace). (b) Spike fired by the master neuron and anticipated spike fired by the slave neuron. The delay $\tau$ is $13 \mathrm{~ms}$.

We have chosen parameters such that in the absence of external stimulus there are no spikes $(T=6 \mathrm{C}$, $T_{o}=10 \mathrm{C}, V_{l}=-75 \mathrm{mV}$, and all other parameters are taken from Refs. $\left.{ }^{17-19}\right)$. The strength of the synaptic connection is $K=0.03 \mathrm{~ms}^{-1}$, the delay time is $\tau=50 \mathrm{~ms}$, and the common external stimulus is a Gaussian white noise which has zero mean and correlation $\left\langle\xi(t) \xi\left(t^{\prime}\right)\right\rangle=2 D \delta\left(t-t^{\prime}\right)$ with $D=0.5(\mu \mathrm{A})^{2} \mathrm{~ms} / \mathrm{cm}^{2}$. Qualitatively the same results are obtained with this model (see Figs. 4c, 4d), indicating that the anticipation 
phenomenon is robust and model independent. In this case anticipated synchrony is also observed in the absence of external stimulus, for parameters such that there is spontaneous regular or irregular spike activity (as in Refs. $\left.{ }^{17-19}\right)$. The slave neuron produces pulses that anticipate those of the master neuron. As in the experiments, the simulations show that the slave system occasionally fires an extra pulse which does not correspond to a pulse fired by the master, and all the pulses fired by the master have the corresponding anticipated pulse fired by the slave. Furthermore, we have also considered different types of random external stimuli (colored noise and a periodic signal with random amplitude) finding the same anticipating behavior. Notice that in the FHN model (Fig. 4b) the anticipation time is less than the duration of the pulse, while in the HH model (Fig. $4 \mathrm{~d}$ ) the anticipation time is much larger than the duration of the pulse. This might be explained by the different location of the fixed points in parameter space. In the FHN model, when the master and the slave neurons are in the unexcited state the coupling is very small because the values of the master and slave fast variables, $x_{m}$ and $x_{s}$, are nearly zero. On the contrary in the $\mathrm{HH}$ model in the unexcited state the coupling is strong (since the membrane potentials of the master and the slave neurons, $V_{m}$ and $V_{s}$, are different from zero). Both, in the FHN and HH models the number of errors increases with the anticipation time (and the larger the value of $\tau$, the larger the strength of the synaptic connection needed to observe synchrony). We have also found that by considering a chain of cascaded slave neurons, the number of errors for a given anticipation time can be reduced. The quantification of the synchronization as well as a detailed study of the parameter region where it occurs will be reported elsewhere.

\section{CONCLUSIONS}

We hope that our findings will stimulate the search for anticipated synchrony in biological systems. Our results show that non-linearity, noise and delayed feedback might conspire to produce new interesting phenomena. We believe that the current work may have applications, for example, by offering an alternative technique to study irregular pulsing behavior in excitable systems such as sensory neurons or cardiac tissue. Furthermore, there are many neural structures, including the thalamus and the visual cortex, where the conditions we have simulated, namely inhibitory delayed feedback, are present. Experiments based on artificial electrical synapses specifically designed to search for anticipated synchrony can be performed. Only the identification and the recording of voltage signals at two points in the neural circuitry are needed (as in the experiments presented in Ref. ${ }^{20}$ ).

\section{ACKNOWLEDGMENTS}

We gratefully thank Dante Chialvo, John Rinzel and Oreste Piro for useful suggestions and a critical reading of the manuscript. C. Masoller is supported by Proyecto de Desarrollo de Ciencias Bsicas, Comisión Sectorial de Investigación Científica, Uruguay, and Universitat de les Illes Balears, Spain. M. Ciszak, O. Calvo, C. Mirasso and R. Toral acknowledge support from MCyT (Spain) through projects CONOCE BFM2000-1108 and SINFIBIO BFM2001-0341-C01-01.

\section{REFERENCES}

1. Gray, C. M. et al. (1989) Nature 338, 334.

2. Singer, W. and Gray C. M. (1995) Annu. Rev. Neurosci. 18, 555.

3. Traub, R. D. et al. Nature (1996) 383, 621.

4. Roelfsema, P. R. (1997) Nature 385, 157.

5. Rao, R. P. N. and Ballard, D. H. Nature Neuroscience (1999) 2, 79.

6. Ballard, D. H. et al. Neurocomputing (2000) 32, 17.

7. Mehta, M. R. Neuroscientist (2001)7, 490-495.

8. Koch, C., Biophysics of Computation (Oxford University Press, New York, 1999).

9. Glass, L. Hunter, P. and McCulloch, A. Eds. Theory of Heart (Springer-Verlag, New York, 1991).

10. Details of the circuits can be found at http://www.imedea.uib.es/ ${ }^{\sim}$ claudio.

11. Linares-Barranco B. et al. (1991) IEEE J. of Solid-State Circuits 28, 956.

12. Voss, H. U. (2001) Phys. Rev. Lett. 87, 014102.

13. Movies of experimental spike trains can be found in the web page: http://www.imedea.uib.es/ ${ }^{\sim}$ claudio 

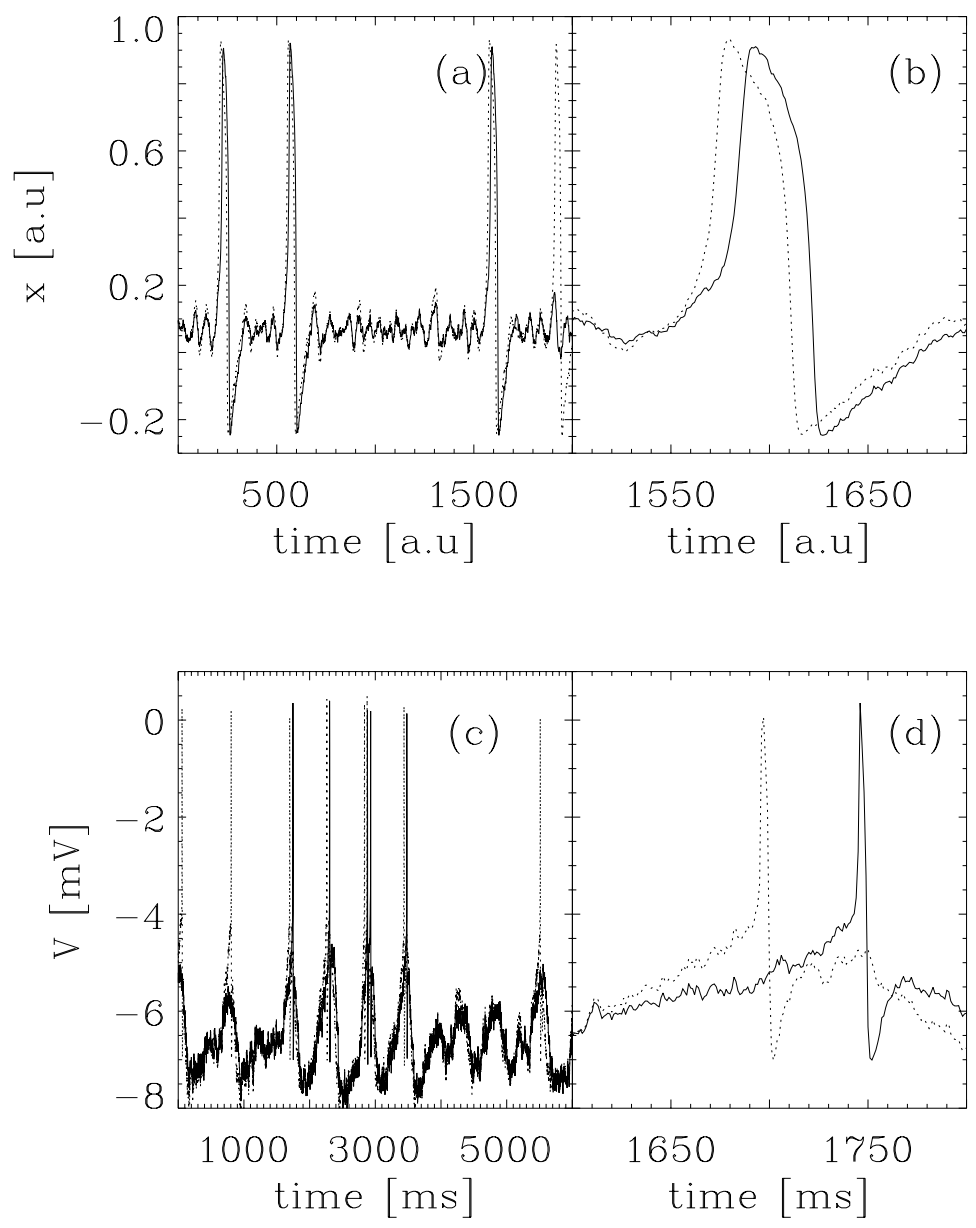

Figure 4. Trains of spikes obtained from numerical simulations of models of unidirectionally coupled neurons. (a), (b) Simulation of two FitzHugh-Nagumo neurons. (c), (d) Simulation of two Hodgkin-Huxley neurons.

14. Voss, H. U. (2000) Phys. Rev. E 61, 5115.

15. Masoller, C. (2001) Phys. Rev. Lett. 86, 2782.

16. Sivaprakasam, S. et al. (2001) Phys. Rev. Lett. 87, 154101; (4 October 2001) Phys. Rev. Focus 8, story 18 .

17. Braun, H. A. et al. (1998) Int. J. of Bifurcation Chaos Appl. Sci. Eng. 8, 881.

18. Feudel, U. et al. (2000) Chaos 10, 231.

19. Braun, W. et al. (2000) Phys. Rev. E 62, 6352.

20. Elson, R. C. et al. (1998) Phys. Rev. Lett. 81, 5692. 